\title{
Coupling of a cardiovascular model with a thermoregulation model to predict human blood pressure under unsteady environmental conditions
}

\author{
Yoshito Takahashi ${ }^{1, *}$, Masayuki Oata ${ }^{1}$, Jun-ichi Asaka ${ }^{2}$, Akihisa Nomoto $^{1}$, and Shin-ichi Tanabe ${ }^{1}$ \\ ${ }^{1}$ Department of Architecture, Waseda University, 3-4-1 Okubo, Shinjuku-ku Tokyo 169-8555, Japan \\ ${ }^{2}$ Department of Architecture, Former Waseda University, 3-4-1 Okubo, Shinjuku-ku Tokyo 169-8555, Japan
}

\begin{abstract}
We coupled a cardiovascular model with a thermoregulation model to predict human blood pressure in unsteady environmental conditions. Our cardiovascular model is a lumped parameter model and consists of 42 segments, which include the entire artery and vein system, divided into 18 segments; the heart, divided into 4 segments; and the pulmonary artery and vein. The vessel parameters were adjusted on the basis of local body blood volume and flow of the thermoregulation model in a thermoneutral environment. Blood pressure under unsteady environmental conditions is predicted by changing the heart rate and vessel resistance of the cardiovascular model which is controlled by blood flow that the thermoregulation model predicts. It is possible to predict the increase in blood pressure under cold environmental conditions and the increase in cardiac output under hot environmental conditions and when bathing. The model was validated by simulating bathing experiments. As the result, the model predicted the peak blood pressure later than the experimental data in a cold environment. To improve the accuracy of the model, it is necessary to consider a method for controlling the heart rate, vessel resistance, and gravity effects after a change in posture.
\end{abstract}

\section{Introduction}

In recent years, a decline in health due to thermal stress while bathing in winter among elderly people is increasing in Japan [1]. The sudden change of a thermal environment in the dressing-room and the bathroom is considered as a cause of bathing accidents. Thus, it is important to predict blood pressure fluctuation under unsteady environments such as bathing for indoor thermal evaluation and measures to prevent a decline in health.

Some research groups $[2,3]$ have tried to couple a thermoregulation model and a cardiovascular model to predict fluctuations in blood pressure due to thermal environmental changes. However, in these researches, both blood flows predicted by the thermoregulation model and the cardiovascular model do not coincide.

In this study, we developed a cardiovascular model coupled with a thermoregulation model called JOS-2 [4] to predict blood pressure fluctuation under unsteady environments and simulated a bathing experiment.

\section{Cardiovascular model}

\subsection{Whole constitution}

Fig. 1. Shows the cardiovascular model, and equations (1) through (3) show the basic functions of the cardiovascular model. Our cardiovascular model is a lumped parameter model and consists of 42 segments, which include the entire artery and vein system, divided into 18 segments; the heart, divided into 4 segments; and the pulmonary artery and vein. The vessel parameters were adjusted on the basis of local body blood volume and flow of the thermoregulation model in a thermoneutral environment.

The blood volume at each segment is calculated by subtracting the dead volume and dividing by the compliance. The segments are connected via a resistance, and the blood flows due to blood pressure differences between the segments. Corresponding to the thermoregulation model, the artery-vein of the systemic circulation was divided by resistance of the core and skin. Hand and Foot segments, which form the end of limbs, are provided Arteriovenous Anastomoses (AVA). Considering check valves, the minimum value of the blood flow in veins, atriums, and ventricles is $0 \mathrm{~mL} / \mathrm{s}$. The change in the blood volume of each segment per unit of time is calculated by the difference between the blood inflow and outflow.

$$
\begin{gathered}
Q=\frac{P_{\text {in }}-P_{\text {out }}}{R} \\
\frac{d V}{d t}=Q_{\text {in }}-Q_{\text {out }} \\
P=\frac{V-V_{d}}{C}
\end{gathered}
$$

\footnotetext{
*Yoshito Takahashi: ytakahashi@tanabe.arch.waseda.ac.jp
} 


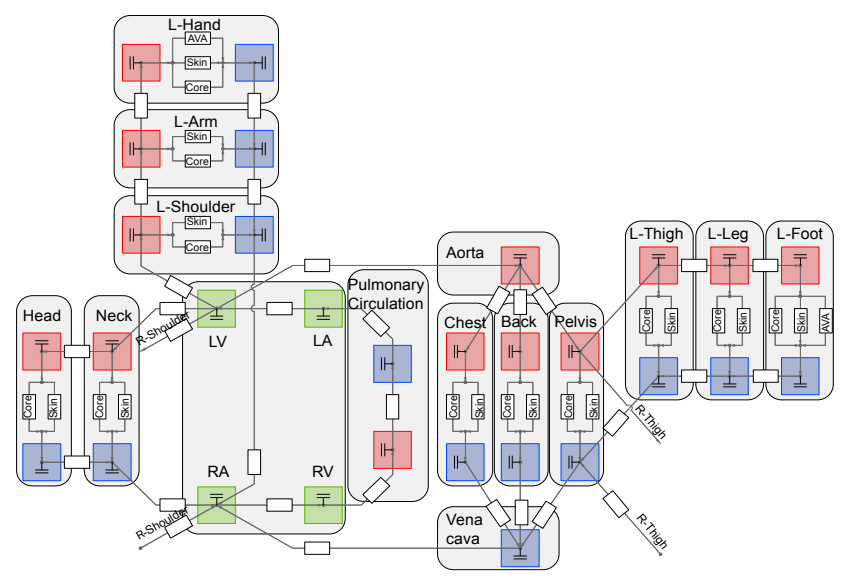

Fig. 1. Cardiovascular model

\subsection{Modeling of the heart}

Equations (4) and (5) show expression of controlling cardiac compliance, and Table 1 shows the physical properties of the heart. The cardiac compliance is periodically changed to simulate contraction and expansion of the heart. Based on the model of Liang [5], the mathematical formula was simplified and used. The contraction start time of ventricles was delayed by 0.2 seconds from the atriums.

Table 1. Property of the heart [5]

\begin{tabular}{|l|c|c|c|c|}
\hline & RA & RV & LA & LV \\
\hline$E_{a}[\mathrm{mmHg} / \mathrm{mL}]$ & 0.06 & 0.55 & 0.07 & 2.75 \\
\hline$E_{b}[\mathrm{mmHg} / \mathrm{mL}]$ & 0.07 & 0.05 & 0.09 & 0.08 \\
\hline$T_{c p}[\mathrm{sec}]$ & 0.17 & 0.30 & 0.17 & 0.30 \\
\hline$T_{r p}[\mathrm{sec}]$ & 0.17 & 0.15 & 0.17 & 0.15 \\
\hline
\end{tabular}

$$
\begin{gathered}
\boldsymbol{C}=\frac{\mathbf{1}}{\boldsymbol{E}_{\boldsymbol{a}} \cdot \boldsymbol{e}(\boldsymbol{t})-\boldsymbol{E}_{\boldsymbol{b}}} \\
e_{(t)}= \begin{cases}0.5 \cdot\left[1-\cos \frac{\pi \cdot t}{T_{c p}}\right] & 0 \leq t<T_{c p} \\
0.5 \cdot\left[1+\cos \frac{\pi \cdot\left(t-T_{c p}\right)}{T_{r p}}\right] & T_{c p} \leq t<+T_{c p}+T_{r p} \\
0 & T_{c p}+T_{r p} \leq t\end{cases}
\end{gathered}
$$

\subsection{Adjusting physical property of the model}

Resistance and compliance were adjusted as the blood flow and blood volume of the cardiovascular model were under thermoneutral environment. Table 2 shows the blood flow and blood volume under a thermoneutral environment. The blood flow and blood volume under a thermoneutral environment were calculated by the thermoregulation model, which was the standard body (74.43 $\mathrm{kg}$ body weight, $1.87 \mathrm{~m}^{2}$ body surface area, male, and 20 years old). Blood volumes of the arteries and veins of the chest, back, and pulmonary circulation; aorta; and vena cava in the cardiovascular model were distributed from the total blood volume of central blood pool and arteries and veins of the chest and back in the thermoregulation model. Fig. 2 shows the relationship between blood volume and pressure [6]. Based on literature, it was assumed that the dead volume of the artery and vein was $85 \%$ of the blood volume. The dead volume of the heart was $0 \mathrm{~mL}$. The whole vascular resistance of the pulmonary circulation was assumed to be $0.10 \mathrm{mmHg} / \mathrm{mL}$, and the whole vascular resistance of systemic circulation was assumed to be $1.05 \mathrm{mmHg} / \mathrm{mL}$. The heart rate at thermal neutral was assumed to be 60 bpm. The cardiac output of the model of Liang is smaller than the cardiac output of thermoregulation model of the standard body. To match cardiac outputs in both models, Eb was multiplied by 0.87 .

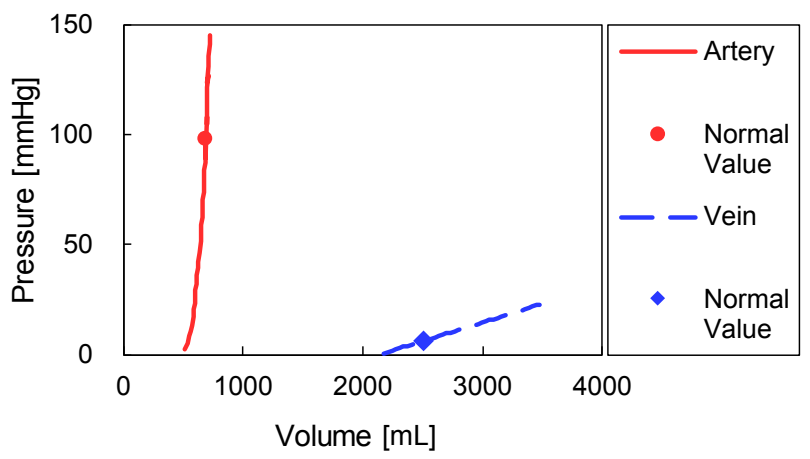

Fig. 2. Relationship between the blood volume and pressure [6]

Table 2. Blood volume and flow of JOS-2 in thermoneutral environment

\begin{tabular}{|c|r|r|r|c|c|}
\hline \multirow{2}{*}{ Segment } & \multicolumn{2}{|c|}{$\begin{array}{c}\text { Blood Volume } \\
{[\mathrm{mL}]}\end{array}$} & \multicolumn{3}{c|}{$\begin{array}{c}\text { Blood Flow } \\
{[\mathrm{mL} / \mathrm{s}]}\end{array}$} \\
\cline { 2 - 7 } & \multicolumn{1}{|c|}{ Artery } & \multicolumn{1}{c|}{ Vein } & \multicolumn{1}{c|}{ Core } & Skin & A VA \\
\hline Head & 85.49 & 285.86 & 10.04 & 0.49 & - \\
\hline Neck & 22.26 & 75.70 & 4.23 & 0.09 & - \\
\hline Chest & 106.86 & 377.59 & 25.29 & 0.55 & - \\
\hline Back & 98.85 & 347.31 & 24.80 & 0.41 & - \\
\hline Pelvis & 235.99 & 740.93 & 10.06 & 0.63 & - \\
\hline L-Shoulder & 16.56 & 63.23 & 0.66 & 0.25 & - \\
\hline L-Arm & 8.10 & 34.73 & 0.34 & 0.14 & - \\
\hline L-Hand & 3.92 & 18.70 & 0.09 & 0.31 & 0.47 \\
\hline R-Shoulder & 16.56 & 63.23 & 0.66 & 0.25 & - \\
\hline R-Arm & 8.10 & 34.73 & 0.34 & 0.14 & - \\
\hline R-Hand & 3.92 & 18.70 & 0.09 & 0.31 & 0.47 \\
\hline L-Thigh & 72.40 & 250.24 & 1.57 & 0.40 & - \\
\hline L-Leg & 35.62 & 133.58 & 0.63 & 0.18 & - \\
\hline L-Foot & 9.17 & 40.07 & 0.05 & 0.26 & 0.39 \\
\hline R-Thigh & 72.40 & 250.24 & 1.57 & 0.40 & - \\
\hline R-Leg & 35.62 & 133.58 & 0.63 & 0.18 & - \\
\hline R-Foot & 9.17 & 40.07 & 0.05 & 0.26 & 0.39 \\
\hline Central & & 1780.19 & - & - & - \\
\hline Blood & & 5529.71 & & & 88.08 \\
\hline Total & & & &
\end{tabular}


Table 3. Blood volume and flow of JOS-2 in a thermoneutral environment

\begin{tabular}{|ll|c|c|c|c|}
\hline & & Pre-room & Dressing room & Bathroom & Dressing room \\
\hline Time & {$[\mathrm{min}]$} & 1025 & 7 & 10 & 10 \\
\hline Metabolic Rate & {$[\mathrm{met}]$} & 0.95 & 0.95 & 0.95 & 0.95 \\
\hline Clothing Insulation & {$[\mathrm{clo}]$} & 0.97 & 0.04 & 0.04 & 0.04 \\
\hline Operative Temp. & {$\left[{ }^{\circ} \mathrm{C}\right]$} & 25 & 10 & 10 & 10 \\
\hline Relative Humidity & {$[\%]$} & 50 & 50 & 50 & 50 \\
\hline Air Velocity & {$[\mathrm{m} / \mathrm{s}]$} & 0.15 & 0.15 & 0.15 & 0.15 \\
\hline
\end{tabular}

\subsection{Coupling of the cardiovascular model with the thermoregulation model}

Blood pressure under unsteady environmental conditions is predicted by changing the heart rate and vessel resistance of the cardiovascular model and controlled by the blood flow that the thermoregulation model predicts. First, blood flow fluctuation accompanying thermal environment change is predicted by the thermoregulation model. Next, skin, AVA blood vessel resistance, and heart rate of the cardiovascular model are controlled so that the blood flow of the cardiovascular model is consistent with the blood flow of the thermoregulation model. It is possible to predict blood pressure increases under cold environmental conditions and cardiac output increases under hot environmental conditions and when bathing.

In the cardiovascular model, difference in blood flow due to different body types is not considered; however, in the thermoregulation model, blood flow is modified by body surface area and body weight. Thus, in this study, the blood flow predicted by the thermoregulation model changes to the blood flow of the standard body.

\section{Simulation}

To verify the accuracy of the model, the experiment on human subjects conducted by Hashiguchi [7] was simulated using our models. Hashiguchi measured the human body physiology when 8 young males were exposed to a cold environment and bathing. The experiments were conducted in the pre-room, dressing room, and bathroom. The pre-room was controlled at $25{ }^{\circ} \mathrm{C}$ air temperature and $50 \%$ relative humidity, the dressing room and bathroom was controlled at $10{ }^{\circ} \mathrm{C}$ air temperature. The experimental schedule is shown below. The subjects were instructed to:

1) Stay in the pre-room for more than 25 minutes wearing a long sleeve shirt and trousers in a sitting position

2) Move to the dressing room and change to clothes of only shorts, stay for 7 minutes in a sitting position

3) Move to the bathroom and take a bath with hot water, $40{ }^{\circ} \mathrm{C}$ temperature, for 10 minutes in a sitting position

4) Move to the dressing room, wipe off the waterdrop with a towel, and stay for 10 minutes in a sitting position
Table 3 shows the input conditions of the environment in the thermoregulation model. The model was analyzed in the same condition of the pre-room for 1000 minutes to set the initial value. Fig. 3 shows the clothing insulation set in this simulation [8]. The insulation of clothes used in the experiment was assumed 0.97 clo in the pre-room and 0.04 clo in the other rooms. Metabolic rate was set at 0.95 met. It was assumed that the body segments other than the head and neck were completely immersed in hot water during bathing, and the maximum value of the skin blood flow was 5 times the basal blood flow based on the measured value of the previous study [9]. The hot water temperature was $40^{\circ} \mathrm{C}$, the convective heat transfer coefficient on the hot water contact surface was 237 $\mathrm{W} /\left(\mathrm{m}^{2} \mathrm{~K}\right)[10]$, and heat transfer by radiation and evaporation did not occur. In consideration of getting wet after bathing, it was assumed that it takes 3 minutes to completely wipe off water drops on the body surface, and the wettedness of body segments in contact with water was set to the upper limit of 0.7 .

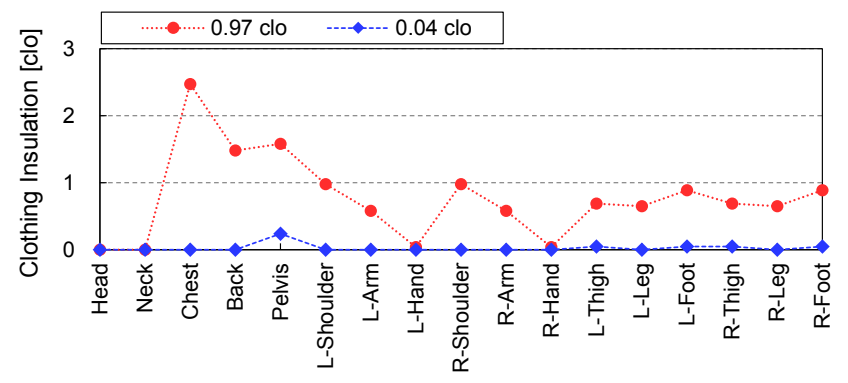

Fig. 3. Clothing insulation [8]

\section{Results}

Fig. 4 shows the results of the simulation and the data of the experiment by Hashiguchi. Experimental values in the figure are average values of 8 subjects, and the simulated blood pressure are values in the L-Shoulder. The mean skin temperature was predicted accurately overall. The difference between the experimental value and the simulated value was large immediately after entering the dressing room and bathing. The simulated mean skin temperature was estimated to be higher than the experimental value at the maximum of $2.6{ }^{\circ} \mathrm{C}$. In the cardiovascular model, an increase in blood pressure at cold exposure and a decrease in blood pressure at bathing were predicted because the heart rate and vessel resistance

\footnotetext{
*Yoshito Takahashi: ytakahashi@tanabe.arch.waseda.ac.jp
} 
were controlled. However, the fluctuation range of the systolic blood pressure due to the change in the thermal environment was predicted to be smaller than the experimental value, and the fluctuation range of the diastolic blood pressure was predicted to be larger than the experimental value. There was also a time lag in the blood pressure peak.
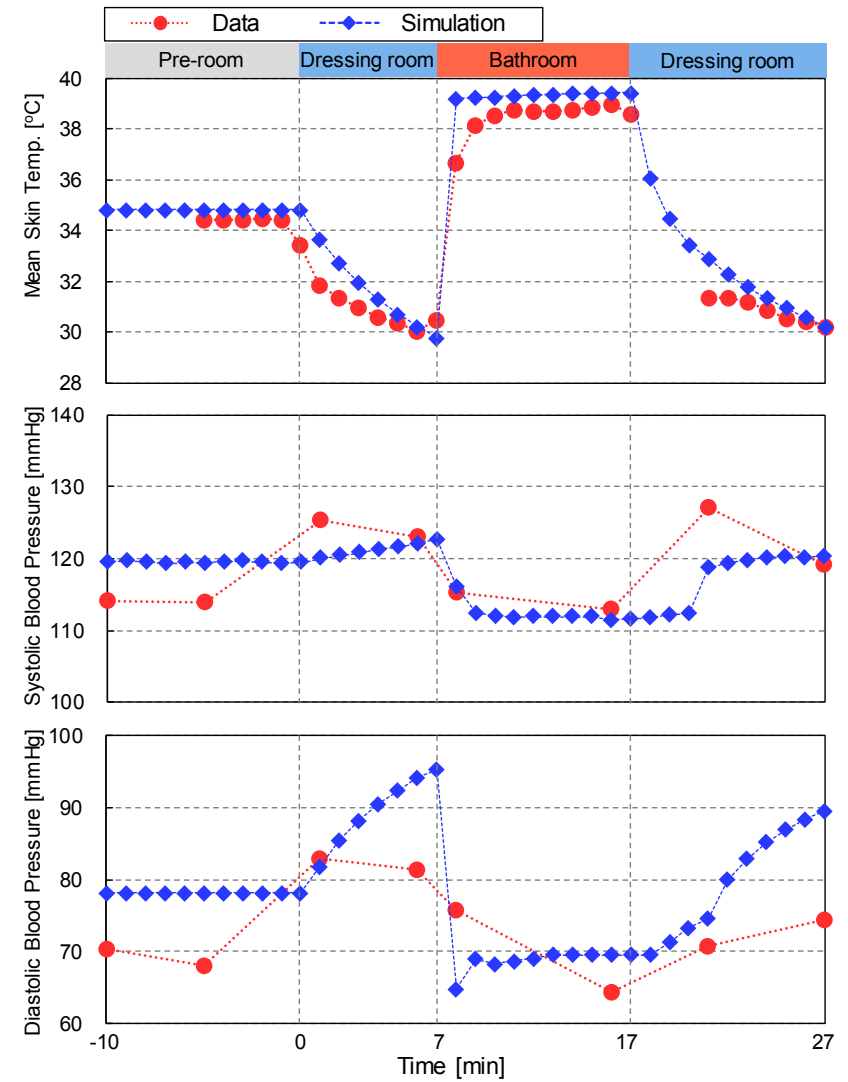

Fig. 4. Results of the simulation and the data of the experiment by Hashiguchi [7]

\section{Discussion}

To improve accuracy after movement between rooms, it is necessary to study the influence of gravity due to posture change and the control method of heart rate and vascular resistance. Hashiguchi's experiments showed that the increase in blood pressure at the time of entering the dressing room was caused not only by an increase in peripheral vascular resistance due to cold exposure but also the influence of walking. In this study, heart rate and vascular resistance of the skin and AVA of the cardiovascular model were changed based on the thermoregulation model. On the other hand, in the actual human body, in addition to the requirement for body temperature control, there is a demand for stability of the cardiovascular system to minimize change in blood pressure [11].

\section{Conclusion}

In this study, we coupled a cardiovascular model with a thermoregulation model to predict human blood pressure under unsteady environmental conditions. Blood pressure in unsteady environmental conditions is predicted by changing the heart rate and vessel resistance of the cardiovascular model and controlled by blood flow predicted by the thermoregulation model. It is possible to predict blood pressure increases under cold environmental conditions and cardiac output increases under hot environmental conditions and when bathing. The model was validated by simulating the bathing experiment. As the result, the model predicted an increase in blood pressure at cold exposure and a decrease in blood pressure at bathing because the heart rate and vessel resistance were controlled. However, the peak of the blood pressure later than the experimental data in a cold environment.

\section{References}

1. S. Hori, Health and Labor Sciences Research Grants (2013)

2. D. Terayama, R. Sugawara, H. Sakamoto, T. Goto, Architectural Institute of Japan, Summaries of technical papers of annual meeting 2017, 245-246

3. N. Masugi, S. Hokoi, Architectural Institute of Japan, Summaries of technical papers of annual meeting 2017, 243-244

4. Y. Kobayahi, S. Tanabe, Build. Environ. 66, 1-10 (2013)

5. F. Y. Liang, S. Takagi, R. Himeno, H. Liu, J. Biomech. 42, 692-704 (2009)

6. I. P. Herman, Physics of the Human Body-Second Edition- (Springer, 2016)

7. N. Hashiguchi, F. Ni, Y. Tochihara, J. Physiol. Anthropol. Appl. Human Sci. 21-6, 277-283 (2002)

8. J. Lee, H. Zhang, E. Arens, Center for the Built Environment (2013)

9. C. Miwa, K. Sugimura, Y. Kawamura, H. Deguchi, S. Iwase, The Japanese Society of Balneology, Climatology and Physical Medicine 65-4, 187-193 (2002)

10. M. Serikawa, M. Mae, Y. Akamine, M. Satoh, S. Iwamoto, T. Kuramuchi, Journal of Environmental Engineering, Architectural Institute of Japan 77, 143-151 (2012)

11. M. Iriki, Thermoregulation Mechanism (Bunkodo, 1995)

\section{Symbol}

$Q, Q_{\text {in }}, Q_{\text {out }}: \quad$ Blood flow $[\mathrm{mL} / \mathrm{s}]$

$V \quad$ : Blood volume [mL]

$V_{d} \quad: \quad$ Dead blood volume $[\mathrm{mL}]$

$P, P_{\text {in }}, P_{\text {out }}:$ Blood pressure $[\mathrm{mmHg}]$

$R \quad: \quad$ Vascular resistance $[\mathrm{mmHg} \cdot \mathrm{s} / \mathrm{mL}]$

C : Vascular compliance $[\mathrm{mL} / \mathrm{mmHg}]$

$t \quad:$ Time [s]

$E_{a}, E_{b} \quad: \quad$ Elanstance $[\mathrm{mmHg} / \mathrm{mL}]$

$e(t) \quad: \quad$ Normalized time-varying elastance [-]

$T_{c p}, T_{r p} \quad: \quad$ Cardiac duration [s] 